\title{
The Association of Dietary Acid Load with Resting Metabolic Rate and Metabolic Components in Overweight and Obese Women: A Cross Sectional Study
}

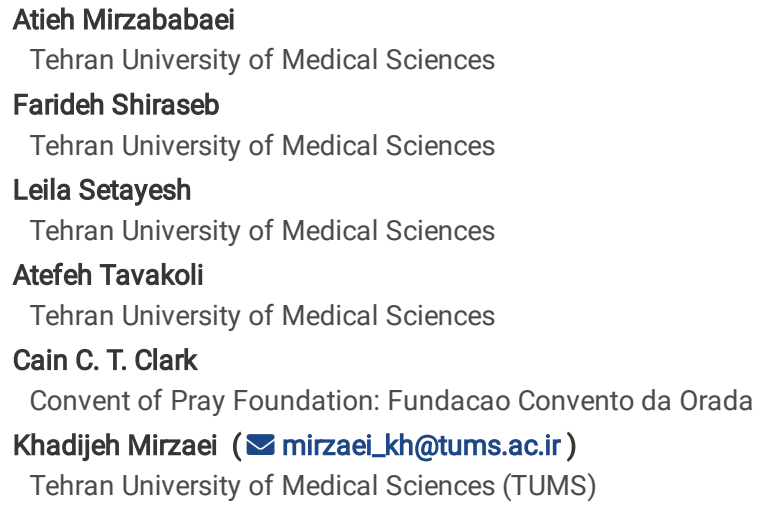

Version of Record: A version of this preprint was published at Clinical Nutrition ESPEN on December 1st, 2021. See the published version at https://doi.org/10.1016/j.clnesp.2021.11.033. 


\section{Abstract}

Objective: Several epidemiologic studies have reported that dietary acid load is associated with metabolic profiles; however, to our knowledge, the relationship of this dietary pattern with resting metabolic rate (RMR) among obese and overweight females remains unreported. Therefore, this study aimed to evaluate the association of dietary acid load RMR and metabolic components among overweight and obese adult women.

Results: It was observed that after correction for potential confounders, DBP and NEAP and PRAL scores were inversely associated (P<0.05). NEAP index was inversely associated with $\mathrm{RMR}(\beta=-0.25,95 \% \mathrm{Cl}=-0.1 .5$ to $2.08, \mathrm{P}=0.02)$, and positively associated with $\mathrm{WC}(\beta=1.009,95 \% \mathrm{Cl}=-1.43$ to $3.45, \mathrm{P}=0.05)$ and $\mathrm{WHR}$ $(\beta=0.01,95 \% \mathrm{Cl}=-0.01$ to0.04, $\mathrm{P}=0.03)$, such that subjects with higher scores in NEAP had lower RMR and higher WC and WHR. We also observed that $D A L$ $(\beta=-0.02,95 \% \mathrm{Cl}=-0.08$ to $0.03, \mathrm{P}=0.08)$ and $\mathrm{PRAL}(\beta=-0.037,95 \% \mathrm{Cl}=-1.05$ to $0.03 \mathrm{P}=0.07)$ were marginally associated with $\mathrm{RMR}$.

\section{Introduction}

The prevalence of obesity are increasing globally, it has been demonstrated that obesity is strongly related to several life-limiting disorders, including cardiovascular diseases and diabetes (1). Obesity is regarded as a may be influenced by eating habits, low energy expenditure(2).

The role of acid-base homeostasis in cardio-metabolic risk factors has recently been posited $(3,4)$. Animal protein such as fish and meatare influential in a higher acid production in the body while, vegetables and fruits are known as alkaline foods $(5,6)$.Dietary patterns have evolved towards a western-style diet, which is defined by a higher intake of animal products, fast foods that result in higher production of acid in body $(4,7)$.

Potential renal acid load (PRAL), net endogenous acid production (NEAP) (8), and dietary acid load )DAL) (9) indexes are commonly used to estimate dietary acid load in epidemiological studies. PRAL and DAL scores are, generally, better predictors of dietary acid load, as, in those formulas, calcium, phosphorus, and magnesium, as well as protein and potassium, are included; while NEAP is calculated, simply, by the ratio of protein and potassium $(5,10)$. Accordingly, a negative PRAL value demonstrates a base forming potential, while a positive value indicates an acid-forming potential (11).

Previous studies have mainly focused on the association of acid/base imbalance on kidney stones and bone health $(12,13)$ metabolic syndrome $(14)$. Metabolic acidosis, which can be influenced by dietary factors (15-17), may adversely affect blood pressure (18-20) through an increase in cortisol production $(15,21,22)$. Higher production of cortisol, as the result of metabolic acidosis $(15,17)$, may also have an adverse influence on the other cardiometabolic risk factors, including hypertension, hypocholesteremia and insulin resistance (IR) $(23,24)$. Indeed, several studies have illustrated that resting metabolic rate (RMR) can be reduced in type 2 diabetes with IR (25).It has been posited that individuals with low RMR are at higher risk of developing obesityrelated disorders(26).

In the present study, we assessed the relationship between three score of dietary acid load and metabolic components and RMR in overweight and obese women.

\section{Materials And Methods Study population}

In the current stud 375 women aged 18 to 48 , with a body mass index (BMI) $\geq 25 \mathrm{~kg} / \mathrm{m}^{2}$, were recruited from Health Centers in Tehran, Iran between $2017-$ 2019. The Medical Research Ethics Committee of Tehran University of Medical Sciences (TUMS) approved the study with the following identification IR.TUMS.VCR.REC.1395.1597. All participants expressed their willingness to participate in the study by providing informed written consent. The overall exclusion criteria were as follows: any history of acute and chronic disease including hypertension, diabetes mellitus, cardiovascular disease, and impaired renal and liver function, as well as regular use of medicine, pregnant or lactating, intake of alcohol, smoking, and menopause. In addition, participants who had been following any arbitrary special dietary regimen, as well as those with chronic disease(s) affecting their diet, or if their daily energy intake was $<800$ $\mathrm{kcal}$ or $>4200 \mathrm{kcal}(27)$,were excluded.

\section{Dietary assessment}

Dietary intake was assessed using a validated semi quantitative food frequency questionnaire (FFQ) with 147- food items(28). All FFQs were completed by a trained dietitian, who asked participants to designate their intake frequency for each food item consumed during the past year, on a daily, weekly, or monthly basis. Finally, we converted portion sizes of foods to grams by using household measures (29).

\section{Assessment of dietary acid load}

Dietary acid-base load was evaluated via 3 indexes: PRAL, NEAP, and DAL which were calculated by using nutrients derived from the FFQ with the following formulas:

1. PRAL $(\mathrm{mEq} / \mathrm{d})=0.4888 \times$ dietary protein $(\mathrm{g} / \mathrm{d})+0.0366 \times$ dietary phosphorus $(\mathrm{mg} / \mathrm{d})-0.0205 \times$ dietary potassium $(\mathrm{mg} / \mathrm{d})-0.0125 \times \mathrm{calcium}(\mathrm{mg} / \mathrm{d})-$ $0.0263 \times$ magnesium $(\mathrm{mg} / \mathrm{d})(30)$

2. NEAP $=54.5 \times$ dietary protein $(\mathrm{g} / \mathrm{d}) /$ dietary potassium $(\mathrm{mg} / \mathrm{d})-10.2(31)$

3. $\mathrm{DAL}(\mathrm{mEq} /$ day $)=\mathrm{PRAL}+\left(\right.$ body surface area $\left(\mathrm{m}^{2}\right) \times 41(\mathrm{mEq} /$ day $\left.) / 1.73 \mathrm{~m}^{2}\right)(32)$

Body surface area (BSA) was calculated using the Du Bois formula: $0.007184 \times$ height $0.725 \times$ weight $0.425(9,33)$. We adjusted both PRAL and NEAP for total energy intake, employing the residual method. 


\section{Anthropometric assessments}

Weight was determined on a digital scale, where participants wore light indoor clothing, without shoes, and was recorded to the nearest $100 \mathrm{~g}$. Height was measured to the nearest $0.5 \mathrm{~cm}$ while participants were in the normal standing position, without shoes. Waist circumference (WC), waist-to-hip ratio (WHR), waist-to-height ratio (WHtR) were measured according to standard procedures(34).

\section{Blood pressure assessment}

Blood pressure measurements were taken using a standard mercury sphygmomanometer.

\section{Physical activity}

The Short form of the International Physical Activity Questionnaire (IPAQ) (35) was applied to evaluate physical activity and according to the frequency and time of common activities of daily life over the past year (36).

\section{Measurement of biochemical parameters}

Fasting serum glucose, insulin, total cholesterol, triglyceride (TG), low density lipoprotein (LDL), high density lipoprotein (HDL) were measured from blood samples drawn after 8-12 h of overnight fasting. All samples were assessed by standard methods at the Nutrition and Biochemistry Laboratory of the school of Nutritional and Dietetics at TUMS. IR was estimated by homeostasis model assessment (HOMA)(37)

\section{Resting metabolic rate assessment}

RMR measurement was performed by professional nutritionists using a standard protocol (38), and was measured by indirect calorimetry (spirometer METALYZERR 3B-R3, Cortex Biophysik GmbH, Leipzig, Germany).

\section{Complete body composition analysis}

Body composition of subjects was evaluated via Body Composition Analyzer BC-418MA- In Body (United Kingdom), in accordance with manufacturer guidelines.

\section{Statistical analysis}

The Kolmogorov-Smirnov test was used to assess the distribution of the data. Baseline characteristics of participants were compared by independent samples $t$ test between the median of PRAL, NEAP, and DAL score and chi square $\left(\chi^{2}\right)$ tests for categorical variables. Also, we adjusted variables for confounders using Analysis of covariance (ANCOVA). General linear regression (GLM) was used to assess the association of scores with body composition, biochemical variables and RMR per kg. Data were analyzed using IBM SPSS version 25.0(SPSS, Chicago, IL, USA) and P-values $\leq 0.05$ were considered for significant.

\section{Result}

\section{Study Population Characteristics}

The mean of quantitative variables including; age, BMI, RMR, of the participants in our study were; 36.67 years $(\mathrm{SD}=9.1), 30.98 \mathrm{~kg} / \mathrm{m}^{2}(\mathrm{SD}=3.9), 1574.96$ $\mathrm{kg} /$ day $(\mathrm{SD}=259.71)$.

\section{Association between quantitative and qualitative variables among acid load scores}

Physical activity was higher in high intake of PRAL, NEAP, DAL and also about age in high intake of NEAP $(P<0.05)$. FFM was higher in high intake of PRAL and DAL $(P<0.05)$ and normal RMR was higher in low intake of NEAP $(P=0.04)($ Table 1$)$. 
Table 1

Study participant characteristics between High and Low acid load scores

\begin{tabular}{|c|c|c|c|c|c|c|c|c|c|c|c|c|c|}
\hline \multirow[t]{3}{*}{ Variables } & \multicolumn{3}{|c|}{ PRAL } & \multirow{3}{*}{$\begin{array}{l}\mathrm{P}- \\
\text { value }\end{array}$} & \multirow{3}{*}{$\begin{array}{l}\text { P- } \\
\text { value }^{\dagger}\end{array}$} & \multicolumn{2}{|l|}{ NEAP } & \multirow{3}{*}{$\begin{array}{l}\mathrm{P} \text { - } \\
\text { value }\end{array}$} & \multirow{3}{*}{$\begin{array}{l}\text { P- } \\
\text { value }^{\dagger}\end{array}$} & \multicolumn{2}{|l|}{ DAL } & \multirow{3}{*}{$\begin{array}{l}\mathrm{P} \text { - } \\
\text { value }\end{array}$} & \multirow{3}{*}{$\begin{array}{l}\text { P- } \\
\text { Ve } \\
t\end{array}$} \\
\hline & \multirow{2}{*}{\multicolumn{2}{|c|}{$\begin{array}{l}\text { Low } \\
(<-19.98)\end{array}$}} & High & & & Low & High & & & Low & High & & \\
\hline & & & $\begin{array}{l}( \\
\geq-19.98)\end{array}$ & & & $(<-9.03)$ & $(\geq-9.03)$ & & & $(<-18.52)$ & $(\geq-18.52)$ & & \\
\hline \multicolumn{2}{|c|}{ Age (years ) } & $35.77 \pm 8.78$ & $\begin{array}{l}37.17 \pm \\
9.25\end{array}$ & 0.14 & 0.29 & $\begin{array}{l}35.44 \pm \\
8.78\end{array}$ & $\begin{array}{l}37.36 \pm \\
9.22\end{array}$ & 0.04 & 0.11 & $\begin{array}{l}35.58 \pm \\
8.71\end{array}$ & $\begin{array}{l}37.24 \pm \\
9.26\end{array}$ & 0.08 & 0 : \\
\hline \multicolumn{2}{|c|}{$\begin{array}{l}\text { Physical } \\
\text { activity } \\
\text { (MET- } \\
\text { minutes/week) }\end{array}$} & $854.07 \pm 919.3$ & $\begin{array}{l}1560.98 \\
\pm \\
2782.47\end{array}$ & $<0.001$ & 0.02 & $\begin{array}{l}88.83 \pm \\
944.30\end{array}$ & $\begin{array}{l}1538.56 \\
\pm \\
2789.83\end{array}$ & 0.01 & 0.04 & $\begin{array}{l}863.00 \pm \\
930.01\end{array}$ & $\begin{array}{l}1530.74 \\
\pm \\
2745.44\end{array}$ & $<0.001$ & 0. \\
\hline \multicolumn{14}{|c|}{ Anthropometric variables } \\
\hline \multicolumn{2}{|l|}{ Weight(kg) } & $\begin{array}{l}79.64 \pm \\
10.36\end{array}$ & $\begin{array}{l}80.64 \pm \\
11.43\end{array}$ & 0.13 & 0.30 & $\begin{array}{l}79.60 \pm \\
10.46\end{array}$ & $\begin{array}{l}80.66 \pm \\
11.37\end{array}$ & 0.36 & 0.78 & $\begin{array}{l}79.64 \pm \\
10.36\end{array}$ & $\begin{array}{l}80.64 \pm \\
11.43\end{array}$ & 0.38 & 0. \\
\hline \multicolumn{2}{|l|}{ Height(cm) } & $\begin{array}{l}161.49 \pm \\
6.08\end{array}$ & $\begin{array}{l}161.06 \pm \\
5.74\end{array}$ & 0.38 & 0.02 & $\begin{array}{l}161.53 \pm \\
6.10\end{array}$ & $\begin{array}{l}161.04 \pm \\
5.74\end{array}$ & 0.42 & 0.21 & $\begin{array}{l}161.43 \pm \\
6.04\end{array}$ & $\begin{array}{l}161.10 \pm \\
5.78\end{array}$ & 0.97 & 0. \\
\hline \multicolumn{2}{|l|}{$\mathrm{HC}(\mathrm{cm})$} & $\begin{array}{l}105.66 \pm \\
6.05\end{array}$ & $\begin{array}{l}105.65 \pm \\
9.16\end{array}$ & 0.99 & 0.83 & $\begin{array}{l}105.65 \pm \\
6.10\end{array}$ & $\begin{array}{l}105.66 \pm \\
9.14\end{array}$ & 0.98 & 0.56 & $\begin{array}{l}105.35 \pm \\
5.71\end{array}$ & $\begin{array}{l}105.82 \pm \\
9.2\end{array}$ & 0.87 & 0 : \\
\hline \multirow[t]{2}{*}{$\begin{array}{l}\text { BMI } \\
\left(\mathrm{kg} / \mathrm{m}^{2}\right)\end{array}$} & Crude & $\begin{array}{l}30.70 \pm \\
3.89\end{array}$ & $\begin{array}{l}31.14 \pm \\
3.90\end{array}$ & \multirow[t]{2}{*}{0.28} & \multirow[t]{2}{*}{0.22} & $\begin{array}{l}30.66 \pm \\
3.86\end{array}$ & $\begin{array}{l}30.16 \pm \\
3.91\end{array}$ & \multirow[t]{2}{*}{0.22} & \multirow[t]{2}{*}{0.30} & $\begin{array}{l}30.64 \pm \\
3.84\end{array}$ & $\begin{array}{l}31.17 \pm \\
3.92\end{array}$ & \multirow[t]{2}{*}{0.19} & \multirow[t]{2}{*}{0 : } \\
\hline & Model 1 & $\begin{array}{l}30.84 \pm \\
0.31\end{array}$ & $\begin{array}{l}30.29 \pm \\
0.32\end{array}$ & & & $\begin{array}{l}30.88 \pm \\
0.31\end{array}$ & $\begin{array}{l}30.33 \pm \\
0.32\end{array}$ & & & $\begin{array}{l}30.79 \pm \\
0.31\end{array}$ & $\begin{array}{l}30.28 \pm \\
0.31\end{array}$ & & \\
\hline \multicolumn{14}{|c|}{ Body composition } \\
\hline $\mathrm{BFM}(\mathrm{kg})$ & Crude & $\begin{array}{l}34.30 \pm \\
8.40\end{array}$ & $\begin{array}{l}34.98 \pm \\
8.95\end{array}$ & 0.45 & 0.13 & $\begin{array}{l}34.14 \pm \\
8.46\end{array}$ & $\begin{array}{l}35.08 \pm \\
9.87\end{array}$ & 0.30 & 0.56 & $\begin{array}{l}33.55 \pm \\
7.44\end{array}$ & $\begin{array}{l}35.37 \pm \\
9.33\end{array}$ & 0.04 & 0.1 \\
\hline & Model 1 & $\begin{array}{l}33.79 \pm \\
0.26\end{array}$ & $\begin{array}{l}33.63 \pm \\
0.27\end{array}$ & & & $34.23 . \pm 0.45$ & $\begin{array}{l}33.19 \pm \\
32.97\end{array}$ & & & $\begin{array}{l}33.78 \pm \\
0.26\end{array}$ & $\begin{array}{l}33.26 \pm \\
0.27\end{array}$ & & \\
\hline FFM(kg) & Crude & $\begin{array}{l}46.51 \pm \\
5.49\end{array}$ & $\begin{array}{l}46.53 \pm \\
5.85\end{array}$ & 0.98 & 0.01 & $\begin{array}{l}46.65 \pm \\
5.61\end{array}$ & $\begin{array}{l}46.45 \pm \\
5.78\end{array}$ & 0.74 & 0.47 & $\begin{array}{l}46.17 \pm \\
5.21\end{array}$ & $\begin{array}{l}45.71 \pm \\
5.96\end{array}$ & 0.37 & 0. \\
\hline & Model 1 & $\begin{array}{l}46.12 \pm \\
0.46\end{array}$ & $\begin{array}{l}47.02 \pm \\
0.47\end{array}$ & & & $\begin{array}{l}46.33 \pm \\
0.45\end{array}$ & $\begin{array}{l}46.81 \pm \\
0.47\end{array}$ & & & $\begin{array}{l}46.10 \pm \\
0.46\end{array}$ & $\begin{array}{l}47.04 \pm \\
0.47\end{array}$ & & \\
\hline $\begin{array}{l}\text { Deviation } \\
\text { from }\end{array}$ & Crude & $\begin{array}{l}-7.65 \pm \\
13.09\end{array}$ & $\begin{array}{l}-9.24 \pm \\
12.1\end{array}$ & 0.27 & 0.06 & $\begin{array}{l}-7.57 \pm \\
12.03\end{array}$ & $\begin{array}{l}-9.32 \pm \\
12.03\end{array}$ & 0.23 & 0.04 & $\begin{array}{l}-7.72 \pm \\
13.09\end{array}$ & $\begin{array}{l}-9.14 \pm \\
0.96\end{array}$ & 0.33 & 0. \\
\hline RMR & Model1" & $\begin{array}{l}-7.40 \pm \\
1.18\end{array}$ & $\begin{array}{l}-9.14 \pm \\
1.23\end{array}$ & & & $-7.37 \pm 1.12$ & $\begin{array}{l}-10.09 \pm \\
1.15\end{array}$ & & & $\begin{array}{l}-7.49 \pm \\
1.12\end{array}$ & $\begin{array}{l}-9.98 \pm \\
1.17\end{array}$ & & \\
\hline Qualitative & variables & & & & & & & & & & & & \\
\hline Economic s & tatus & & & 0.42 & 0.31 & & & 0.46 & 0.69 & & & 0.34 & 0. \\
\hline Low level & & $34(38.6)$ & $54(61.4)$ & & & $32(36.4)$ & $56(63.6)$ & & & $33(37.5)$ & $55(62.5)$ & & \\
\hline Moderate le & & 72(39.1) & $112(60.9)$ & & & 72(39.1) & $112(60.9)$ & & & 70(38) & $114(62)$ & & \\
\hline High level & & $35(31.8)$ & $75(68.2)$ & & & $35(31.8)$ & $75(68.2)$ & & & $33(30)$ & 77(70) & & \\
\hline Education le & evel & & & & & & & & & & & 0.73 & 0. \\
\hline Illiterate & & $25(1)$ & $3(75)$ & 0.84 & 0.48 & $1(25)$ & $3(75)$ & 0.74 & 0.68 & $1(25)$ & $3(75)$ & & \\
\hline Under diplor & & $17(34.7)$ & $32(65.3)$ & & & 16(32.7) & $33(67.3)$ & & & 16(32.7) & $33(67.3)$ & & \\
\hline Diploma & & $54(35.3)$ & $99(64.7)$ & & & $54(35.3)$ & $99(64.7)$ & & & 51(33.3) & 102(66.7) & & \\
\hline Master and & higher & 73(38.6) & $116(61.4)$ & & & $74(39.2)$ & $115(60.8)$ & & & 72(38.1) & 117(61.9) & & \\
\hline Marital stat & & & & 0.40 & 0.69 & & & 0.10 & 0.17 & & & 0.29 & 0. \\
\hline Single & & 35)32.1 ( & 74)67.9 ( & & & $33(30.3)$ & 76(69.7) & & & $34(31.2)$ & $75(68.8)$ & & \\
\hline Married & & ${ }^{110) 38.5}$ & 176)61.5 & & & $112(39.2)$ & $174(60.8)$ & & & 106(37.1) & $180(62.9)$ & & \\
\hline
\end{tabular}




\begin{tabular}{|c|c|c|c|c|c|c|c|c|c|c|c|c|}
\hline \multirow[t]{3}{*}{ Variables } & \multicolumn{2}{|l|}{ PRAL } & \multirow{3}{*}{$\begin{array}{l}\mathrm{P}- \\
\text { value }\end{array}$} & \multirow{3}{*}{$\begin{array}{l}\text { P- } \\
\text { value }^{\dagger}\end{array}$} & \multicolumn{2}{|l|}{ NEAP } & \multirow{3}{*}{$\begin{array}{l}\mathrm{P} \text { - } \\
\text { value }\end{array}$} & \multirow{3}{*}{$\begin{array}{l}\text { P- } \\
\text { value }^{\dagger}\end{array}$} & \multicolumn{2}{|l|}{ DAL } & \multirow{3}{*}{$\begin{array}{l}\mathrm{P} \text { - } \\
\text { value }\end{array}$} & \multirow{3}{*}{$\begin{array}{l}\text { P. } \\
\text { Ve } \\
t\end{array}$} \\
\hline & Low & High & & & Low & High & & & Low & High & & \\
\hline & $(<-19.98)$ & $\left(\begin{array}{l}(19.98) \\
\geq\end{array}\right.$ & & & $(<-9.03)$ & $(\geq-9.03)$ & & & $(<-18.52)$ & $(\geq-18.52)$ & & \\
\hline
\end{tabular}

HC, Hip circumference; BMI, body mass index, BFM, body fat mass; FFM, fat free mass; PRAL, potential renal acid load; DAL, dietary acid load NEAP, net endogenous acid production

Values are mean \pm SD for crude model and mean \pm SE for adjusted model and qualitative variables are presented as $\mathrm{n}(\%)$.

All variables adjusted with age, energy intake, physical activity, BMI, economic status

Model1|l: further adjustment for fat free mass

Crude P-value obtained from independent sample T test.

t adjust P-value obtained from analysis of covariance

\section{Association between some dietary intake components among acid load scores}

After adjustment for confounders, cereals consumption was higher in high intake of all three scores (PRAL, NEAP, DAL) and fruits consumption was higher in low intake of all three scores $(\mathrm{P}<0.001)$; vegetables consumption was higher in low PRAL and DAL scores, and fat intake was higher in high PRAL, NEAP, and DAL scores (Table 2). 
Table 2

Dietary intakes of study population between high and low acid load Scores

\begin{tabular}{|c|c|c|c|c|c|c|c|c|c|}
\hline \multirow[t]{2}{*}{ Variables } & \multicolumn{2}{|l|}{ PRAL } & \multirow{2}{*}{$\begin{array}{l}\text { P- } \\
\text { value }^{\dagger}\end{array}$} & \multicolumn{2}{|l|}{ NEAP } & \multirow{2}{*}{$\begin{array}{l}\text { P- } \\
\text { value }^{\dagger}\end{array}$} & \multicolumn{2}{|l|}{ DAL } & \multirow{2}{*}{$\begin{array}{l}\text { P- } \\
\text { value }^{\dagger}\end{array}$} \\
\hline & Low $(<-19.98)$ & $\begin{array}{l}\text { High ( } \\
\geq-19.98 \text { ( }\end{array}$ & & Low $(<-9.03)$ & High ( $\geq-9.03)$ & & $\begin{array}{l}\text { Low } \\
(<-18.52)\end{array}$ & $\begin{array}{l}\text { High ( } \\
\geq-18.52)\end{array}$ & \\
\hline \multicolumn{10}{|l|}{ Food groups } \\
\hline Cereal(g/d) & $\begin{array}{l}1258.95 \pm \\
16.39\end{array}$ & $\begin{array}{l}1357.70 \pm \\
16.87\end{array}$ & $\hat{0.001}$ & $\begin{array}{l}1256.23 \pm \\
16.23\end{array}$ & $\begin{array}{l}1361.47 \pm \\
16.89\end{array}$ & $\hat{0} .001$ & $\begin{array}{l}1258.95 \pm \\
16.39\end{array}$ & $\begin{array}{l}1357.70 \pm \\
16.87\end{array}$ & <. 001 \\
\hline Fruits $(g / d)$ & $\begin{array}{l}1676.69 \pm \\
22.31\end{array}$ & $\begin{array}{l}652.15 \pm \\
23.89\end{array}$ & <. 001 & $\begin{array}{l}1668.69 \pm \\
22.42\end{array}$ & $\begin{array}{l}1395.42 \pm \\
23.28\end{array}$ & <. 001 & $\begin{array}{l}1677.69 \pm \\
22.31\end{array}$ & $\begin{array}{l}1390.6 \pm \\
22.78\end{array}$ & < 0.001 \\
\hline Vegetables $(\mathrm{g} / \mathrm{d})$ & $497.74 \pm 2012$ & $\begin{array}{l}294.69 \pm \\
19.59\end{array}$ & <. 001 & $\begin{array}{l}305.59 \pm \\
19.84\end{array}$ & $487 \pm 20.55$ & <. 001 & $\begin{array}{l}1215.59 \pm \\
19.79\end{array}$ & $1013 \pm 20.15$ & < 0.001 \\
\hline Legumes $(g / d)$ & $263.40 \pm 3.78$ & $\begin{array}{l}261.41 \pm \\
3.88\end{array}$ & 0.52 & $\begin{array}{l}264.23 \pm \\
3.57\end{array}$ & $260.49 \pm 3.70$ & 0.47 & $\begin{array}{l}263.30 \pm \\
3.60\end{array}$ & $261.52 \pm 3.68$ & 0.73 \\
\hline $\operatorname{Nuts}(g / d)$ & $144.98 \pm 1.37$ & $\begin{array}{l}138.81 \pm \\
1.41\end{array}$ & $<0.001$ & $\begin{array}{l}144.09 \pm \\
1.38\end{array}$ & $139.72 \pm 1.43$ & 0.03 & $\begin{array}{l}145.30 \pm \\
11.18\end{array}$ & $\begin{array}{l}138.53 \pm \\
18.59\end{array}$ & $<0.001$ \\
\hline Dairy $(g / d)$ & $\begin{array}{l}1272.09 \pm \\
21.60\end{array}$ & $\begin{array}{l}1234.64 \pm \\
21.32\end{array}$ & 0.26 & $\begin{array}{l}1272.09 \pm \\
20.66\end{array}$ & $\begin{array}{l}1244.64 \pm \\
21.37\end{array}$ & 0.36 & $\begin{array}{l}1260.49 \pm \\
20.90\end{array}$ & $\begin{array}{l}1257.09 \pm \\
22.02\end{array}$ & 0.97 \\
\hline $\operatorname{Eggs}(g / d)$ & $19.77 \pm 1.28$ & $23.94 \pm 1.31$ & 0.02 & $19.11 \pm 1.26$ & $24.68 \pm 19.11$ & 0.003 & $19.92 \pm 1.29$ & $23.74 \pm 1.31$ & 0.04 \\
\hline Meat ${ }^{\wedge}(g / d)$ & $\begin{array}{l}350.42 .39 \pm \\
4.53\end{array}$ & $\begin{array}{l}366.12 \pm \\
4.66\end{array}$ & 0.01 & $\begin{array}{l}349.40 \pm \\
4.66\end{array}$ & $367.36 \pm 4.66$ & 0.007 & $\begin{array}{l}351.43 \pm \\
4.57\end{array}$ & $364.95 \pm 4.66$ & 0.04 \\
\hline \multicolumn{10}{|c|}{ Energy and macronutrients } \\
\hline Energy (kcal) & $\begin{array}{l}2515.59 \pm \\
66.65\end{array}$ & 2703.51 .45 & 0.02 & $\begin{array}{l}2530.33 \pm \\
66.75\end{array}$ & $\begin{array}{l}2694.62 \pm \\
51.52\end{array}$ & 0.05 & $\begin{array}{l}2491.56 \pm \\
67.66\end{array}$ & $\begin{array}{l}2713.20 \pm \\
50.81\end{array}$ & $<0.001$ \\
\hline Carbohydrates $(\mathrm{g} / \mathrm{d})$ & $\begin{array}{l}370.85 \pm \\
42.57\end{array}$ & $\begin{array}{l}330.47 \pm \\
46.58\end{array}$ & <. 001 & $\begin{array}{l}369.86 \pm \\
3.81\end{array}$ & $331.67 \pm 3.96$ & <. 001 & $\begin{array}{l}370.77 \pm \\
3.83\end{array}$ & $331.37 \pm 3.91$ & <. 001 \\
\hline Fat $(\mathrm{g} / \mathrm{d})$ & $99.93 \pm 0.51$ & $\begin{array}{l}112.69 \pm \\
0.39\end{array}$ & <. 001 & $\begin{array}{l}100.55 \pm \\
1.71\end{array}$ & $112.12 \pm 1.77$ & <. 001 & $99.86 \pm 0.53$ & $112.65 \pm 0.39$ & <. 001 \\
\hline protein $(\mathrm{g} / \mathrm{d})$ & $92.00 \pm 0.21$ & $93.28 \pm 0.16$ & 0.56 & $91.40 \pm 0.21$ & $93.94 \pm 0.16$ & 0.25 & $92.00 \pm 0.21$ & $93.28 \pm 0.16$ & 0.13 \\
\hline Total fiber(g/d) & $44.32 \pm 1.35$ & $49.40 \pm 1.04$ & 0.05 & $43.83 \pm 1.22$ & $47.13 \pm 1.23$ & 0.06 & $\begin{array}{l}44.100 \pm \\
1.25\end{array}$ & $46.76 \pm 1.22$ & 0.13 \\
\hline $\operatorname{MUFA}(\mathrm{g} / \mathrm{d})$ & $33.98 \pm 0.71$ & $30.87 \pm 0.55$ & <. 001 & $33.72 \pm 0.72$ & $29.20 \pm 0.73$ & 0.001 & $33.25 \pm 0.75$ & $29.82 \pm 0.73$ & 0.001 \\
\hline PUFA(g/d) & $30.87 \pm 0.55$ & $21.87 \pm 0.62$ & $<0.001$ & $21.58 \pm 0.64$ & $18.60 \pm 0.64$ & $<0.001$ & $21.18 \pm 0.66$ & $19.09 \pm 0.64$ & 0.02 \\
\hline $\operatorname{SFA}(g / d)$ & $27.51 \pm 0.47$ & $29.97 \pm 0.61$ & <. 001 & $29.69 \pm 0.63$ & $26.87 \pm 0.64$ & $<0.001$ & $30.09 \pm 0.64$ & $26.60 \pm 0.63$ & $\hat{0} .001$ \\
\hline \multicolumn{10}{|c|}{ Micronutrients and caffeine } \\
\hline $\operatorname{Iron}(\mathrm{mg} / \mathrm{d})$ & $18.88 \pm 1.52$ & $31.30 \pm 1.17$ & 0.09 & $18.60 \pm 0.24$ & $18.82 \pm 0.25$ & 0.53 & $18.48 \pm 0.25$ & $18.93 \pm 0.24$ & 0.20 \\
\hline Zinc (mg/d) & $12.70 \pm 0.21$ & $13.84 \pm 0.16$ & 0.08 & $12.74 \pm 0.19$ & $13.11 \pm 0.19$ & 0.17 & $12.71 \pm 0.19$ & $13.13 \pm 0.19$ & 0.13 \\
\hline Calcium (mg/d) & $\begin{array}{l}1151.11 \pm \\
32.71\end{array}$ & $\begin{array}{l}1339.63 \pm \\
25.29\end{array}$ & 0.19 & $\begin{array}{l}1234.98 \pm \\
25.38\end{array}$ & $\begin{array}{l}1205.600 \pm \\
25.66\end{array}$ & 0.05 & $\begin{array}{l}1153.14 \pm \\
26.10\end{array}$ & $\begin{array}{l}1185.86 \pm \\
25.44\end{array}$ & 0.37 \\
\hline Magnesium(mg/d) & $504.41 \pm 6.14$ & $428 \pm 7.96$ & $\hat{0} .001$ & $\begin{array}{l}490.45 \pm \\
6.84\end{array}$ & $428.44 \pm 6.77$ & <. 001 & $\begin{array}{l}489.47 \pm \\
6.75\end{array}$ & $427.19 \pm 6.93$ & <. 001 \\
\hline Potassium (mEq/d) & $\begin{array}{l}4931.33 \pm \\
63.14\end{array}$ & $\begin{array}{l}2499.4 \pm \\
209.9\end{array}$ & $\dot{0} 001$ & $\begin{array}{l}4993.33 \pm \\
67.53\end{array}$ & $\begin{array}{l}3690.11 \pm \\
66.79\end{array}$ & 0.001 & $\begin{array}{l}4959.74 \pm \\
67.68\end{array}$ & $\begin{array}{l}3677.52 \pm \\
69.43\end{array}$ & <. 001 \\
\hline Sodium (mg/d) & $\begin{array}{l}4347.68 \pm \\
108.48\end{array}$ & 4852.55 & 0.18 & $\begin{array}{l}4360.44 \pm \\
98.48\end{array}$ & $\begin{array}{l}4679.05 \pm \\
102.48\end{array}$ & 0.02 & $\begin{array}{l}4353.21 \pm \\
9.16\end{array}$ & $\begin{array}{l}4681.28 \pm \\
101.69\end{array}$ & 0.02 \\
\hline Phosphor (mg/d) & $\begin{array}{l}1633.98 \pm \\
25.98\end{array}$ & $\begin{array}{l}1700.7 \pm \\
20.07\end{array}$ & 0.68 & $\begin{array}{l}1624.61 \pm \\
25.42\end{array}$ & $\begin{array}{l}1658.69 \pm \\
25.70\end{array}$ & 0.35 & $\begin{array}{l}1637.81 \pm \\
26.04\end{array}$ & $\begin{array}{l}1644.95 \pm \\
25.38\end{array}$ & 0.84 \\
\hline Vitamin $C(m g / d)$ & $216.63 \pm 6.19$ & $\begin{array}{l}141.18 \pm \\
8.01\end{array}$ & <. 001 & $250.43 v 8.38$ & $145.16 \pm 8.29$ & $\begin{array}{l}< \\
0.001\end{array}$ & $250.17 \pm 8.2$ & $141.57 \pm 8.43$ & $\hat{0} .001$ \\
\hline Vitamin $E(m g / d)$ & $18.38 \pm 0.68$ & $16.23 \pm 0.52$ & 0.24 & $18.36 \pm 0.73$ & $16.51 \pm 0.74$ & 0.08 & $17.85 \pm 0.75$ & $17.05 \pm 0.74$ & 0.45 \\
\hline Vitamin A (mg/d) & $\begin{array}{l}887.79 \pm \\
30.03\end{array}$ & $\begin{array}{l}673.3 \pm \\
29.70\end{array}$ & $<.001$ & $\begin{array}{l}888.46 \pm \\
29.94\end{array}$ & $\begin{array}{l}672.71 \pm \\
29.61\end{array}$ & $\hat{0.001}$ & $\begin{array}{l}879.99 \pm \\
29.67\end{array}$ & $\begin{array}{l}673.67 \pm \\
29.67\end{array}$ & $\hat{0} .001$ \\
\hline Thiamin $(\mathrm{mg} / \mathrm{d})$ & $2.18 \pm 0.04$ & $2.12 \pm 0.03$ & 0.001 & $2.00 \pm 0.03$ & $2.18 \pm 0.2$ & $\hat{0} .001$ & $2.22 \pm 0.02$ & $2.17 \pm 0.03$ & $\hat{0} .001$ \\
\hline
\end{tabular}




\begin{tabular}{|c|c|c|c|c|c|c|c|c|c|}
\hline \multirow[t]{2}{*}{ Variables } & \multicolumn{2}{|l|}{ PRAL } & \multirow{2}{*}{$\begin{array}{l}\text { P- } \\
\text { value }^{\dagger}\end{array}$} & \multicolumn{2}{|l|}{ NEAP } & \multirow{2}{*}{$\begin{array}{l}\text { P- } \\
\text { value }^{\dagger}\end{array}$} & \multicolumn{2}{|l|}{ DAL } & \multirow{2}{*}{$\begin{array}{l}\text { P- } \\
\text { value }\end{array}$} \\
\hline & Low $(<-19.98)$ & $\begin{array}{l}\text { High ( } \\
\geq-19.98 \text { ( }\end{array}$ & & Low $(<-9.03)$ & High ( $\geq-9.03)$ & & $\begin{array}{l}\text { Low } \\
(<-18.52)\end{array}$ & $\begin{array}{l}\text { High ( } \\
\geq-18.52)\end{array}$ & \\
\hline Riboflavin (mg/d) & $2.18 \pm 0.04$ & $2.33 \pm 0.03$ & 0.44 & $2.17 \pm 0.04$ & $2.25 \pm 0.04$ & 0.23 & $2.19 \pm 0.04$ & $2.22 \pm 0.04$ & 0.64 \\
\hline Niacin $(\mathrm{mg} / \mathrm{d})$ & $38.59 \pm 0.52$ & $39.54 \pm 0.40$ & 0.94 & $38.33 \pm 0.50$ & $40.21 \pm 0.51$ & 0.01 & $38.67 \pm 0.52$ & $39.83 \pm 0.51$ & 0.14 \\
\hline Vitamin B6(mg/d) & $2.04 \pm 0.03$ & $2.29 \pm 0.02$ & $\hat{0} .001$ & $2.07 \pm 0.03$ & $2.27 \pm 0.03$ & $\hat{0} .001$ & $2.04 \pm 0.03$ & $2.29 \pm 0.03$ & <. 001 \\
\hline Folate (mcg/d) & $648.23 \pm 9.10$ & $\begin{array}{l}612.54 \pm \\
7.03\end{array}$ & $<0.001$ & $\begin{array}{l}643.00 \pm \\
8.98\end{array}$ & $617.42 \pm 8.99$ & 0.06 & $\begin{array}{l}648.71 \pm \\
9.06\end{array}$ & $612.39 \pm 8.83$ & 0.01 \\
\hline $\begin{array}{l}\text { Vitamin } \\
\text { B12(mcg/d) }\end{array}$ & $13.50 \pm 0.18$ & $14.00 \pm 0.14$ & 0.06 & $13.50 \pm 0.18$ & $14.01 \pm 0.18$ & 0.05 & $13.52 \pm 0.18$ & $13.98 \pm 0.17$ & 0.09 \\
\hline Caffeine (mg/d) & $\begin{array}{l}162.93 \pm \\
16.07\end{array}$ & $\begin{array}{l}315.66 \pm \\
53.44\end{array}$ & $<0.001$ & $\begin{array}{l}161.72 \pm \\
13.52\end{array}$ & $\begin{array}{l}205.25 \pm \\
14.10\end{array}$ & 0.02 & $\begin{array}{l}163.14 \pm \\
13.67\end{array}$ & $\begin{array}{l}203.03 \pm \\
14.02\end{array}$ & 0.04 \\
\hline \multicolumn{10}{|c|}{$\begin{array}{l}\text { MUFA, monounsaturated fatty acid; PUFA, polyunsaturated fatty acid; PRAL, potential renal acid load; DAL, dietary acid load NEAP, net endogenous acid } \\
\text { production }\end{array}$} \\
\hline \multicolumn{10}{|c|}{ Values are mean $\pm S E$. Nutrients and food groups were adjusted for age, BMI, physical activity and total energy intake. } \\
\hline \multicolumn{10}{|c|}{ ^: Meat alternatives included meat, poultery and sea foods. } \\
\hline
\end{tabular}

\section{Relationship of body composition, blood parameters, RMR and acid load scores}

We observed a positive association for NEAP with WC $(P=0.05)$, WHR $(P=0.03)$, TG $(P=0.01)$, also, hs-CRP was positively associated with DAL score $(P \leq$ 0.05). A negative association between NEAP and RMR per $\mathrm{kg}$ was found ( $\beta=-0.66,95 \% \mathrm{Cl}=-1.37$ to $-0.04, \mathrm{P}=0.05)$ in crude model, and in the final model this association became stronger $(\beta=-0.25,95 \% \mathrm{Cl}=-0.1 .5$ to $2.08, P=0.02)$, and a marginal negative association for $P R A L(\beta=-0.03,95 \% \mathrm{Cl}=-1.05$ to $0.03, P=$ $0.07)$ and $\mathrm{DAL}(\beta=-0.02,95 \% \mathrm{Cl}=-0.08$ to $0.03, \mathrm{P}=0.08)$ was found (Table 3$)$. 
Table 3

Association between body composition, blood parameters and all RMR per $\mathrm{kg}(\mathrm{kcal} / \mathrm{d})$ between high and low acid load scores

\begin{tabular}{|c|c|c|c|c|c|c|c|c|c|c|c|c|}
\hline \multirow[t]{2}{*}{ Variables } & & \multicolumn{2}{|l|}{ PRAL } & \multirow{2}{*}{$\begin{array}{l}\mathrm{P} \text { - } \\
\text { value }\end{array}$} & \multirow{2}{*}{$\begin{array}{l}\text { P- } \\
\text { value* }\end{array}$} & \multicolumn{2}{|l|}{ NEAP } & \multirow{2}{*}{$\begin{array}{l}\mathrm{P}- \\
\text { value }\end{array}$} & \multirow{2}{*}{$\begin{array}{l}\text { P- } \\
\text { value* }\end{array}$} & \multicolumn{2}{|l|}{ DAL } & \multirow{2}{*}{$\begin{array}{l}\mathrm{P} \text { - } \\
\text { value }\end{array}$} \\
\hline & & $\beta$ & $\mathrm{Cl}(95 \%)$ & & & $\beta$ & $\mathrm{Cl}(95 \%)$ & & & $\beta$ & $\mathrm{Cl}(95 \%)$ & \\
\hline \multicolumn{13}{|l|}{ Body composition } \\
\hline \multirow[t]{3}{*}{ WC(cm) } & Crude & 0.28 & $-1.66,2.22$ & 0.77 & - & -1.71 & $-4.12,0.69$ & 0.16 & - & -0.03 & $-0.08,01$ & 0.19 \\
\hline & Model 1 & -0.02 & $-0.04,0.004$ & - & 0.10 & -1.09 & $-3.39,1.21$ & - & 0.35 & -1.04 & $-3.40,1.31$ & - \\
\hline & Model 2 & 0.04 & $-1.31,0.04$ & - & 0.33 & 1.009 & $-1.43,3.45$ & - & 0.05 & -0.05 & $-0.13,0.02$ & - \\
\hline \multirow[t]{3}{*}{$\mathrm{NC}(\mathrm{cm})$} & Crude & -1.26 & $-3.26,0.73$ & 0.21 & & -1.12 & $-4.23,1.20$ & 0.18 & - & -1.20 & $-2.61,0.92$ & - \\
\hline & Model 1 & -0.03 & $-0.09,0.03$ & - & 0.34 & -1.35 & $-4.68,1.96$ & - & 0.42 & -1.49 & $-3.91,0.93$ & - \\
\hline & Model 2 & -0.17 & $-0.40,0.05$ & - & 0.13 & -5.95 & $-13.46,1.56$ & - & 0.12 & -0.14 & $-0.36,0.08$ & - \\
\hline \multirow[t]{3}{*}{ WHR } & Crude & -1.07 & $-0.03,0.03$ & 0.98 & - & -0.13 & $-1.51,1.23$ & 0.84 & - & 0.00 & $-0.032,0.03$ & 0.99 \\
\hline & Model 1 & 0.00 & $1.001,0.000$ & - & 0.03 & -0.00 & $-0.02,0.00$ & - & 0.17 & -0.008 & $-0.02,0.00$ & - \\
\hline & Model 2 & 0.001 & $-0.001,0.01$ & - & 0.72 & 0.01 & $-0.01,0.04$ & - & 0.03 & 0.001 & $-0.001,-0.00$ & - \\
\hline \multirow[t]{3}{*}{ WHtR } & Crude & 0.004 & $-0.008,0.01$ & 0.24 & - & -0.01 & $-0.02,0.003$ & 0.11 & - & 0.001 & $0.00-0.004$ & 0.29 \\
\hline & Model 1 & 0.00 & $0.001-0.00$ & - & 0.09 & -0.007 & $-0.02,0.007$ & - & 0.29 & -0.008 & $-0.02,0.00$ & - \\
\hline & Model 2 & 0.00 & $-0.001,0.00$ & - & 0.60 & 0.002 & $-0.01,0.01$ & - & 0.72 & 0.001 & $-0.001,0.00$ & - \\
\hline \multicolumn{13}{|l|}{ Biochemical variables } \\
\hline \multirow[t]{3}{*}{ FBS (mg/dL) } & Crude & 0.04 & $-0.02,0.1$ & 0.19 & - & 2.84 & $0.29,5.38$ & 0.02 & - & 0.03 & $-0.02,0.09$ & 0.24 \\
\hline & Model 1 & 0.06 & $-0.004,0.12$ & - & 0.05 & 2.89 & $0.27,5.51$ & - & 0.03 & 0.06 & $-0.003,0.12$ & - \\
\hline & Model 2 & 0.92 & $0.08,1.76$ & - & 0.03 & 3.82 & $-1.96,9.60$ & - & 0.01 & 0.93 & $0.10,1.76$ & - \\
\hline \multirow[t]{3}{*}{ Insulin (mIU/ ml) } & Crude & 0.001 & $\begin{array}{l}- \\
-0.001,0.002\end{array}$ & 0.41 & - & 0.02 & $-0.03,0.087$ & 0.40 & - & 0.001 & $-0.001,0.002$ & 0.58 \\
\hline & Model 1 & 0.001 & $-0.02,0.00$ & - & 0.24 & 0.01 & $-0.05,0.04$ & - & 0.51 & 0.01 & $-0.05,1.01$ & - \\
\hline & Model 2 & 0.004 & $-0.01,0.00$ & - & 0.07 & 0.01 & $0.00,0.16$ & - & 0.09 & 0.001 & $-0.006,0.001$ & - \\
\hline \multirow[t]{3}{*}{ HOMA-IR } & Crude & 0.004 & $-0.004,0.01$ & 0.28 & - & 0.21 & $-0.12,0.56$ & 0.21 & - & 0.004 & $-0.004,0.01$ & 0.29 \\
\hline & Model 1 & 0.007 & $-0.006,0.03$ & - & 0.17 & 0.19 & $-0.14,0.52$ & - & 0.26 & 0.007 & $-0.001,0.016$ & - \\
\hline & Model 2 & 0.046 & $-0.06,0.15$ & - & 0.02 & 0.13 & $-0.90,0.64$ & - & 0.09 & 0.043 & $-0.06,0.15$ & - \\
\hline \multirow{3}{*}{$\begin{array}{l}\text { Total } \\
\text { cholesterol(mg/dL) }\end{array}$} & Crude & 3.69 & $-5.13,12.51$ & 0.41 & - & 2.47 & $-7.20,12.15$ & 0.61 & - & 0.08 & $-0.13,0.31$ & 0.44 \\
\hline & Model 1 & 0.003 & $-0.24,0.24$ & - & 0.98 & 0.86 & $-8.89,10.61$ & - & 0.17 & 0.56 & $-9.02,10.15$ & - \\
\hline & Model 2 & 0.69 & $-1.51,0.13$ & - & 0.10 & 2.086 & $-24.98,20.81$ & - & 0.85 & -0.59 & $-1.34,0.15$ & - \\
\hline \multirow[t]{3}{*}{ TG(mg/dL) } & Crude & 0.12 & $-0.24,0.50$ & 0.50 & - & 6.18 & $-9.77,22.13$ & 0.76 & - & 0.13 & $-0.23,0.49$ & 0.48 \\
\hline & Model 1 & 0.004 & $-0.42,0.43$ & - & 0.98 & 4.11 & $-12.93,21.16$ & - & 0.04 & -9.61 & $-26.48,7.25$ & - \\
\hline & Model 2 & -0.093 & $-1.58,1.40$ & - & 0.90 & 26.96 & $-14.05,67.99$ & - & 0.01 & 0.09 & $-1.23,1.42$ & - \\
\hline \multirow[t]{3}{*}{$\mathrm{HDL}$ (mg/dL) } & Crude & -2.26 & $-4.93,0.40$ & 0.09 & - & -1.67 & $-4.56,1.21$ & 0.25 & - & -0.029 & $-0.09,0.04$ & 0.40 \\
\hline & Model 1 & -0.03 & $-0.10,0.04$ & - & 0.42 & -1.34 & $-4.35,1.65$ & - & 0.37 & -1.060 & $-4.67,1.47$ & - \\
\hline & Model 2 & -0.02 & $-0.28,0.23$ & - & -0.20 & -1.32 & $-8.48,5.82$ & - & 0.71 & -0.04 & $-0.28,0.19$ & - \\
\hline \multirow[t]{3}{*}{ LDL (mg/dL) } & Crude & 1.28 & $-0.62,3.19$ & 0.18 & - & -2.62 & $-9.07,3.82$ & 0.42 & - & 0.009 & $-0.14,0.16$ & 0.90 \\
\hline & Model 1 & -0.01 & $-0.17,0.15$ & - & 0.88 & -1.67 & $-8.14,4.78$ & - & 0.60 & -0.01 & $-6.53,6.50$ & - \\
\hline & Model 2 & 0.136 & $-7.00,042$ & - & 0.63 & -6.73 & $-22.25,8.78$ & - & 0.39 & 0.08 & $-0.42,0.59$ & - \\
\hline Hs-CRP(mg/l) & Crude & -0.88 & $-2.05,0.27$ & 0.13 & - & -1.10 & $-2.35,0.13$ & 0.08 & - & -0.02 & $-0.05,0.00$ & 0.13 \\
\hline & Model 1 & 0.01 & $-0.04,0.01$ & - & 0.36 & -0.94 & $-2.30,0.41$ & - & 0.17 & 0.72 & $2.03,0.57$ & - \\
\hline & Model 2 & 1.08 & $-0.22,1.10$ & - & 0.06 & 2.76 & $-5.93,0.41$ & - & 0.08 & 0.11 & $-0.21,0.16$ & - \\
\hline SBP (mmHg) & Crude & -0.02 & $-0.10,0.06$ & 0.60 & - & -1.46 & $-5.03,2.09$ & 0.41 & - & -0.02 & $-0.10,0.05$ & 0.59 \\
\hline
\end{tabular}




\begin{tabular}{|c|c|c|c|c|c|c|c|c|c|c|c|c|}
\hline \multirow[t]{2}{*}{ Variables } & & \multicolumn{2}{|l|}{ PRAL } & \multirow{2}{*}{$\begin{array}{l}\text { P- } \\
\text { value }\end{array}$} & \multirow{2}{*}{$\begin{array}{l}\text { P- } \\
\text { value }\end{array}$} & \multicolumn{2}{|l|}{ NEAP } & \multirow{2}{*}{$\begin{array}{l}P- \\
\text { value }\end{array}$} & \multirow{2}{*}{$\begin{array}{l}\text { P- } \\
\text { value* }\end{array}$} & \multicolumn{2}{|l|}{ DAL } & \multirow{2}{*}{$\begin{array}{l}\mathrm{P} \text { - } \\
\text { value }\end{array}$} \\
\hline & & $\beta$ & $\mathrm{Cl}(95 \%)$ & & & $\beta$ & $\mathrm{Cl}(95 \%)$ & & & $\beta$ & $\mathrm{Cl}(95 \%)$ & \\
\hline & Model 1 & -0.05 & $-0.14,0.03$ & - & 0.19 & -2.00 & $-5.60,1.60$ & - & 0.27 & -0.05 & $-0.14,0.02$ & - \\
\hline & Model 2 & -0.11 & $-0.42,0.19$ & - & 0.47 & 0.29 & $-7.75,8.35$ & - & 0.94 & -0.05 & $-0.33,0.23$ & - \\
\hline \multirow[t]{3}{*}{$\mathrm{DBP}(\mathrm{mmHg})$} & Crude & -0.03 & $-0.09,0.01$ & 0.17 & - & -2.07 & $-4.56,0.41$ & 0.10 & - & -0.03 & $-0.09,0.01$ & 0.18 \\
\hline & Model 1 & 0.04 & $-0.11,0.06$ & - & 0.12 & 1.12 & $-4.71,1.22$ & - & 0.09 & 0.01 & $-0.10,-0.11$ & - \\
\hline & Model 2 & 0.33 & $-0.47,-1.15$ & & 0.04 & 0.64 & $-4.99,6.28$ & - & 0.02 & 0.37 & $-0.42,1.18$ & - \\
\hline \multirow{4}{*}{$\begin{array}{l}\text { RMR per kg } \\
\text { (kcal/day) }\end{array}$} & Crude & -0.57 & $-1.27,013$ & 0.11 & - & -0.66 & $-1.37-0.04$ & 0.05 & - & -0.009 & $-0.02,0.00$ & 0.34 \\
\hline & Model 1 & -0.135 & $-0.96,0.69$ & - & 0.74 & -0.28 & $-1.109,0.53$ & - & 0.08 & -0.31 & $-1.13,0.50$ & - \\
\hline & Model2\# & -0.338 & $\begin{array}{l}-1.118- \\
0.442\end{array}$ & - & 0.39 & -0.44 & $-1.21,-0.32$ & - & 0.04 & -0.33 & $-1.11,0.44$ & - \\
\hline & Model3\& & -0.037 & $-1.05,0.03$ & - & 0.07 & -0.25 & $-0.1 .5,2.08$ & - & 0.02 & -0.02 & $-0.08,0.03$ & - \\
\hline
\end{tabular}

HC, Hip circumference; WC, Waist circumference; NC, neck circumference; WHtR, Waist to height ratio; WHR, Waist to hip ratio; HDL, high density lipoproteincholesterol; LDL, low density lipoprotein- cholesterol; TG, Triglycerides; FBS, Fasting blood sugar; HOMA, Homeostatic model assessment, RMR, Resting metc rate, BSA, Body surface area; resting metabolic rate; PRAL, potential renal acid load; DAL, dietary acid load NEAP, net endogenous acid production, SBP, Systo blood pressure; DBP, Diastolic blood pressure; AST, Aspartate Aminotransferase, ALT, Alanine Transferase, Hs-CRP, high-sensitivity C-reactive protein, RQ, respiratory quotient

Values are mean \pm SD for crude model and mean \pm SE for adjusted model.

Model 1: Adjusted for: age, BMI, physical activity, total energy intake, economic status

Model 2: Additionally controlled for significant dietary intakes in Table 2. For PRAL, we controlled the effect cereal, fruits, vegetables, nuts, egg, meat, carbohy total fat intake, phosphor, total fiber, MUFA, PUFA, SFA, caffeine and some of micronutrients magnesium, potassium, vitamin C,A, B1, B6, B9 and B12, NEAP w controlled for calcium, sodium, B3 apart from iron. For Dal score we controlled for all confounder for NEAP apart from B12.

Model2\#: Adjusted for: age, BMI, physical activity and total energy intake and fat free mass

Model3\&: Model2\# confounder variables additionally controlled for significant dietary intakes in Table 2. For PRAL, we controlled the effect cereal, fruits, vegetables, nuts, egg, meat, carbohydrate, total fat intake, phosphor, total fiber, MUFA, PUFA, SFA, caffeine and some of micronutrients magnesium, potassiur vitamin C,A, B1, B6, B9 and B12, NEAP we controlled for calcium, sodium, B3 apart from iron. For Dal score we controlled for all confounder for NEAP apart fr B12.

Crude $\mathrm{p}$-value for all variables obtained from independent sample T test.

*P-value obtained from adjustment. All of p-values obtained from analysis of General linear model.

Definition of RMR per kg:

\section{Discussion}

The results of this study showed a significant association between NEAP intake and RMR, TG. In addition, a favorable relationship was observed between HOMA-IR and PRAL, as well as DAL

Diet acid load scores have been predicted and validated in people with kidney diseases (39), however, to date, studies have reported conflicting findings regarding the relationship of dietary acid load and cardio-metabolic risk factors $(11,14,39-41)$. Indeed, several studies suggested that higher acid load has a negative association $(14,39,40)$, while others refuted this association $(11,39)$.

The current study showed a significant association of NEAP and PRAL with DBP. Studies have reported that in metabolic acidosis related to diet, blood pressure was increased $(18,20)$. The Nurses' Health Study II, which was conducted on 1136 women, showed that a higher NEAP is connected with a greater risk of hypertension (39). However, this relationship is not universally supported; for instance, it has been reported that no association between blood pressure and dietary acid load was evident among participants that were higher than 55 years old (11). Confounding the equivocal reports, the mechanism underlying the proposed relationship have not been well defined, although several pathways have been suggested, such as reduction in elimination of citrate, higher production of cortisol, and higher excretion of calcium $(15,21,42)$.

The association of glycemic profile and dietary acid load has been investigated in several studies. In the present study, we showed that subjects with higher dietary acid score had greater HOMA-IR. It was reported that all of metabolic acidosis markers, such as low serum bicarbonate, higher anion gap, and low urine $\mathrm{pH}$ are associated with insulin resistance $(14,43)$ Sakhaee, et al suggested that subjects with a lower urinary pH had greater prevalence of glucose intolerance and type 2 diabetes in comparison with normal counterparts (12).Several studies have reported that an acidogenic diet may contribute to IR by constantly inducing a chronic acidosis condition in body $(44,45)$. It is also proposed that insulin tendency to bind to its receptors is lessened in metabolic acidosis, which results in IR, albeit in animal studies (46). Moreover, insulin discharge is reduced whenever the body is faced with acid/base variations (47). Another possible mechanism may be related to the association of greater dietary acid load with higher discharge of magnesium, which decreases insulin sensitivity (48).

The associations of urinary acidosis with biochemical parameters have been evaluated in several studies, yet, in spite of controversial results among such studies, we showed a positive association with dietary acid load and TG, WC, and WHR, and a negative relationship with RMR among the subjects. The

Page $9 / 12$ 
influence of an acidogenic diet on RMR can be the result of higher IR (45). In a cohort of 782 adults, reported that RMR and IR were significantly related (49), thus, it can be inferred that higher acid load, by increasing the IR, may reduce RMR. This finding may be suggested as an effective impact of a diet with a lower acid load in women with higher than normal BMI (39). Berg et al did not report any meaningful correlation between urinary acidosis and TG, HDL, and $\mathrm{HbA1c}(4)$.

The association of the dietary acid load and metabolic factors has not been fully discerned, therefore, the underlying mechanisms of how an acidogenic diet influences metabolic outcomes have not been well explained. As mentioned above, animal protein in the diet may represent a cause of increasing acid load in body. The current study focused on overweight and obese women. In addition, the validation of dietary acid load measurement methods among obese and overweight subjects is important; it has been shown that higher protein intake is not the only cause of higher acid production (50). Indeed, in the present study, we also observed that higher consumption of cereals, sodium, fat, and energy intake is related to higher acid load (50).

\section{Conclusion}

We found that dietary acid load scores were significantly related to lower RMR and higher WC, WHR, DBP and HOMA-IR in overweight and obese women.

\section{Limitation}

The present study was conducted on only one gender, so cannot be extrapolated to males. The number of participants were relatively low, moreover, based on the cross-sectional design of the study, we are not able to show any causal inference. Another limitation for assessing dietary intakes from FFQ is misclassification. Albeit we controlled for the effect of the potential confounder by the statistical methods, because of unknown confounder cannot be excluded residual confounding will affect.

\section{Abbreviations}

\section{ARIC}

Atherosclerosis Risk in Communities,B:beta, BIA:Bioelectrical Impedance Analyzer, BMI:Body mass index, BIA:Bioelectrical impedance analysis, BSA:Body surface area, BW:body weight, BFM:body fat mass, Cls:con-fidence intervals, CVD:cardiovascular disease, DAL:dietary acid load, DBP:diastolic blood pressure, FBS:fasting blood sugar ,FFM:Fat Free Mass, GLM:General linear regression, HbA1C:Hemoglobin A1C, HC:hip circumferences, HDL:High density lipoprotein cholesterol, HOMA-IR:Homeostatic model assessment insulin resistance, hs-CRP:Hypersensitive C - reactive protein, IR:insulin resistance, IPAQ:International Physical Activity Questionnaire, LDL:Low density lipoprotein cholesterol, PRAL:potential renal acidload, NC:Neck circumference, NEAP:net endogenous acid production, SDs:Standard Deviations, SEs:standard Errors, TC:total cholesterol, TG:Triglyceride, TUMS:Tehran university of medical science, WC:Waist circumference, WHR:waist and hip, WHtR:waist-to-height ratio

\section{Declarations}

\section{Ethics approval and consent to participate}

The study protocol has approved by the ethics committee of Tehran University of Medical Sciences (TUMS) with the following identification: IR.TUMS.VCR.REC.1395.1597. All methods were carried out in accordance with relevant guidelines and regulations. Each participant was completely informed about the study protocol and provided a written and informed consent form before taking part in the study

\section{Consent for publication}

Not applicable

\section{Availability of data and materials}

The data that support the findings of this study are available from Khadijeh Mirzaei but restrictions apply to the availability of these data, which were used under license for the current study, and so are not publicly available. Data are however available from the authors upon reasonable request and with permission of Khadijeh Mirzaei.

\section{Competing interests}

The authors declare that they have no competing interests.

\section{Funding}

This study was supported by Tehran University of Medical Sciences (Grant number 95-04-161-33893).

\section{Authors' contributions}

The project was designed and implemented by AM and KhM. Data were analyzed and interpreted FSH. LS, AM, AT and CC prepared the manuscript. KhM, supervised overall project.

\section{Acknowledgments}


We thank the school of Nutritional and Dietetics at Tehran University of medical sciences (TUMS) and participants in this investigation.

\section{References}

1. Hurt RT, Kulisek C, Buchanan LA, McClave SA. The obesity epidemic: challenges, health initiatives, and implications for gastroenterologists. Gastroenterology hepatology. 2010;6(12):780.

2. Rahmani A, Sayehmiri K, Asadollahi K, Sarokhani D, Islami F, Sarokhani M. Investigation of the prevalence of obesity in Iran: a systematic review and meta-analysis study. Acta Medica Iranica. 2015:596-607.

3. Adeva MM, Souto G. Diet-induced metabolic acidosis. Clinical nutrition. 2011;30(4):416-21.

4. van den Berg E, Engberink MF, Brink EJ, van Baak MA, Joosten MM, Gans RO, et al. Dietary acid load and metabolic acidosis in renal transplant recipients. Clin J Am Soc Nephrol. 2012;7(11):1811-8.

5. Remer T, Manz F. Potential renal acid load of foods and its influence on urine pH. J Am Diet Assoc. 1995;95(7):791-7.

6. GONICK HC, Goldberg G, Mulcare D. Reexamination of the acid-ash content of several diets. Am J Clin Nutr. 1968;21(9):898-903.

7. Sebastian A, Frassetto LA, Sellmeyer DE, Merriam RL, Morris RC Jr. Estimation of the net acid load of the diet of ancestral preagricultural Homo sapiens and their hominid ancestors. Am J Clin Nutr. 2002;76(6):1308-16.

8. Frassetto LA, Lanham-New SA, Macdonald HM, Remer T, Sebastian A, Tucker KL, et al. Standardizing terminology for estimating the diet-dependent net acid load to the metabolic system. J Nutr. 2007;137(6):1491-2.

9. Verbraecken J, Van de Heyning P, De Backer W, Van Gaal L. Body surface area in normal-weight, overweight, and obese adults. A comparison study. Metabolism. 2006;55(4):515-24.

10. Remer T, Manz F. Estimation of the renal net acid excretion by adults consuming diets containing variable amounts of protein. Am $\mathrm{J}$ Clin Nutr. 1994;59(6):1356-61.

11. Engberink MF, Bakker SJ, Brink EJ, van Baak MA, van Rooij FJ, Hofman A, et al. Dietary acid load and risk of hypertension: the Rotterdam Study. Am J Clin Nutr. 2012;95(6):1438-44.

12. Sakhaee K, Adams-Huet B, Moe OW, Pak CY. Pathophysiologic basis for normouricosuric uric acid nephrolithiasis. Kidney international. 2002;62(3):9719.

13. Reddy ST, Wang C-Y, Sakhaee K, Brinkley L, Pak CY. Effect of low-carbohydrate high-protein diets on acid-base balance, stone-forming propensity, and calcium metabolism. Am J Kidney Dis. 2002;40(2):265-74.

14. Zhang L, Curhan GC, Forman JP. Diet-dependent net acid load and risk of incident hypertension in United States women. Hypertension. 2009;54(4):751-5.

15. Maurer M, Riesen W, Muser J, Hulter HN, Krapf R. Neutralization of Western diet inhibits bone resorption independently of K intake and reduces cortisol secretion in humans. American Journal of Physiology-Renal Physiology. 2003;284(1):F32-40.

16. Remer T, Pietrzik K, Manz F. Short-term impact of a lactovegetarian diet on adrenocortical activity and adrenal androgens. The Journal of Clinical Endocrinology Metabolism. 1998;83(6):2132-7.

17. Sicuro A, Mahlbacher K, Hulter HN, Krapf R. Effect of growth hormone on renal and systemic acid-base homeostasis in humans. American Journal of Physiology-Renal Physiology. 1998;274(4):F650-F7.

18. Resnick LM, Gupta RK, Sosa RE, Corbett ML, Laragh JH. Intracellular pH in human and experimental hypertension. Proceedings of the National Academy of Sciences. 1987;84(21):7663-7.

19. Sharma AM, Kribben A, Schattenfroh S, Cetto C, Distler A. Salt sensitivity in humans is associated with abnormal acid-base regulation. Hypertension. 1990;16(4):407-13.

20. Sharma AM, Cetto C, Schorr U, Spies K-P, Distler A. Renal acid-base excretion in normotensive salt-sensitive humans. Hypertension. 1993;22(6):884-90.

21. Cappuccio FP, Kalaitzidis R, Duneclift S, Eastwood JB. Unravelling the links between calcium excretion, salt intake, hypertension, kidney stones and bone metabolism. J Nephrol. 2000;13(3):169-77.

22. Oshima T, Young EW, editors. Systemic and cellular calcium metabolism and hypertension. Seminars in nephrology; 1995.

23. Dimitriou T, Maser-Gluth C, Remer T. Adrenocortical activity in healthy children is associated with fat mass. Am J Clin Nutr. 2003;77(3):731-6.

24. Fraser R, Ingram MC, Anderson NH, Morrison C, Davies E, Connell JM. Cortisol effects on body mass, blood pressure, and cholesterol in the general population. Hypertension. 1999;33(6):1364-8.

25. Weyer C, Bogardus C, Pratley RE. Metabolic factors contributing to increased resting metabolic rate and decreased insulin-induced thermogenesis during the development of type 2 diabetes. Diabetes. 1999;48(8):1607-14.

26. Ruige JB, Ballaux DP, Funahashi T, Mertens IL, Matsuzawa Y, Van Gaal LF. Resting metabolic rate is an important predictor of serum adiponectin concentrations: potential implications for obesity-related disorders-. Am J Clin Nutr. 2005;82(1):21-5.

27. Fung TT, Hu FB, Pereira MA, Liu S, Stampfer MJ, Colditz GA, et al. Whole-grain intake and the risk of type 2 diabetes: a prospective study in men. Am J Clin Nutr. 2002;76(3):535-40.

28. Toorang F, Sasanfar B, Esmaillzadeh A, Ebrahimpour-Koujan S, Zendehdel K. Comparison of validity of the Food Frequency Questionnaire and the Diet History Questionnaire for assessment of energy and nutrients intakes in an Iranian population. La Revue de Santé de la Méditerranée orientale.26(92020):1062.

29. Ghaffarpour M, Houshiar-Rad A, Kianfar H. The manual for household measures, cooking yields factors and edible portion of foods. Tehran: Nashre Olume Keshavarzy. 1999;7:213. 
30. Remer T, Dimitriou T, Manz F. Dietary potential renal acid load and renal net acid excretion in healthy, free-living children and adolescents. Am J Clin Nutr. 2003;77(5):1255-60.

31. Frassetto LA, Todd KM, Morris RC Jr, Sebastian A. Estimation of net endogenous noncarbonic acid production in humans from diet potassium and protein contents. Am J Clin Nutr. 1998;68(3):576-83.

32. Chamberlain P, Manning F, Morrison I, Harman C, Lange I. Ultrasound evaluation of amniotic fluid volume: I. The relationship of marginal and decreased amniotic fluid volumes to perinatal outcome. American journal of obstetrics gynecology. 1984;150(3):245-9.

33. Du Bois D. A formula to estimate the approximate surface area if height and weight be known. Nutrition. 1989;5:303-13.

34. Fitch KV, Stanley TL, Looby SE, Rope AM, Grinspoon SK. Relationship between neck circumference and cardiometabolic parameters in HIV-infected and non-HIV-infected adults. Diabetes Care. 2011;34(4):1026-31.

35. Craig CL, Marshall AL, Sjöström M, Bauman AE, Booth ML, Ainsworth BE, et al. International physical activity questionnaire: 12-country reliability and validity. Medicine science in sports exercise. 2003;35(8):1381-95.

36. Naderyan S, Sahaf R, Akbari Kamrani AA, Abolfathi Momtaz Y, Ghasemzadeh H, Papi S. Physical activity among Iranian former sportsmen and athletes as possible evidence for continuity theory of aging. Iranian Rehabilitation Journal. 2019;17(2):141-8.

37. Matthews DR, Hosker J, Rudenski A, Naylor B, Treacher D, Turner R. Homeostasis model assessment: insulin resistance and $\beta$-cell function from fasting plasma glucose and insulin concentrations in man. diabetologia. 1985;28(7):412-9.

38. Mirzaei K, Hossein-Nezhad A, Chamari M, Shahbazi S. Evidence of a role of ANGPTL6 in resting metabolic rate and its potential application in treatment of obesity. Minerva Endocrinol. 2011;36(1):13-21.

39. Murakami K, Sasaki S, Takahashi Y, Uenishi K. Association between dietary acid-base load and cardiometabolic risk factors in young Japanese women. British journal of nutrition. 2008;100(3):642-51.

40. Fagherazzi G, Vilier A, Bonnet F, Lajous M, Balkau B, Boutron-Ruault M-C, et al. Dietary acid load and risk of type 2 diabetes: the E3N-EPIC cohort study. Diabetologia. 2014;57(2):313-20.

41. Krupp D, Johner SA, Kalhoff H, Buyken AE, Remer T. Long-term dietary potential renal acid load during adolescence is prospectively associated with indices of nonalcoholic fatty liver disease in young women. J Nutr. 2012;142(2):313-9.

42. Taylor EN, Mount DB, Forman JP, Curhan GC. Association of prevalent hypertension with 24-hour urinary excretion of calcium, citrate, and other factors. American journal of kidney diseases. 2006;47(5):780-9.

43. Crawford SO, Hoogeveen RC, Brancati FL, Astor BC, Ballantyne CM, Schmidt MI, et al. Association of blood lactate with type 2 diabetes: the Atherosclerosis Risk in Communities Carotid MRI Study. Int J Epidemiol. 2010;39(6):1647-55.

44. Cameron MA, Maalouf NM, Adams-Huet B, Moe OW, Sakhaee K. Urine composition in type 2 diabetes: predisposition to uric acid nephrolithiasis. J Am Soc Nephrol. 2006;17(5):1422-8.

45. Souto G, Donapetry C, Calvino J, Adeva MM. Metabolic acidosis-induced insulin resistance and cardiovascular risk. Metabolic Syndrome Related Disorders. 2011;9(4):247-53.

46. Whittaker J, Cuthbert C, Hammond V, Alberti K. The effects of metabolic acidosis in vivo on insulin binding to isolated rat adipocytes. Metab Clin Exp. 1982;31(6):553-7.

47. Rebolledo OR, Hernandez RE, Zanetta AC, Gagliardino J. Insulin secretion during acid-base alterations. American Journal of Physiology-Endocrinology Metabolism. 1978;234(4):E426.

48. Rylander R, Remer T, Berkemeyer S, Vormann Jr. Acid-base status affects renal magnesium losses in healthy, elderly persons. J Nutr. $2006 ; 136(9): 2374-7$.

49. Drabsch T, Holzapfel C, Stecher L, Petzold J, Skurk T, Hauner H. Associations between C-reactive protein, insulin sensitivity, and resting metabolic rate in adults: A mediator analysis. Front Endocrinol. 2018;9:556.

50. Remer T. Influence of nutrition on acid-base balance-metabolic aspects. Eur J Nutr. 2001;40(5):214-20. 